\title{
Identification of genes and transcription factors associated with glucocorticoid response in lens epithelial cells
}

\author{
DING ZHOU ${ }^{1}$, YI ZHANG ${ }^{1}$, LISHAN WANG ${ }^{2}$, YUNDUAN SUN ${ }^{1}$ and PING LIU ${ }^{1}$ \\ ${ }^{1}$ Department of Ophthalmology, The First Affiliated Hospital of Harbin Medical University, Harbin, Heilongjiang 150001; \\ ${ }^{2}$ Bio-X Center, Shanghai Jiao Tong University, Shanghai 200230, P.R. China
}

Received November 5, 2013; Accepted December 12, 2014

DOI: $10.3892 / \mathrm{mmr} .2015 .3308$

\begin{abstract}
Prolonged glucocorticoids (GCs) treatment may lead to the formation of posterior subcapsular cataracts. The present study aimed to investigate differential gene expression in lens epithelial cells (LECs) in response to GCs using DNA microarray profiling. The gene expression profile of GSE13040 was downloaded from the Gene Expression Omnibus database, which includes 12 human LECs treated with vehicle or dexamethasone (Dex) for 4 or $16 \mathrm{~h}$ with six samples at each time period, of which three samples were treated with vehicle (control group) and three samples were treated with Dex (Dex group) at each time point. The differentially expressed genes (DEGs) were identified between the control group and the Dex group at each time period with the thresholds of $\mathrm{P}<0.05$ and $\mid \log \mathrm{FCl}>1$. The DEGs were further analyzed using bioinformatics methods. Firstly, DEGs were subject to a hierarchical cluster analysis. Subsequently, the functional enrichment analysis was performed for the common DEGs between the two time periods. Finally, the transcription factors and binding sites of DEGs associated with response to GC stimulus were analyzed. A total of 696 and 949 DEGs were identified at $4 \mathrm{~h}$ and $16 \mathrm{~h}$, respectively. Hierarchical cluster analysis revealed that DEG expression was higher in the Dex group than in the control group $(\mathrm{P}<0.05)$. A total of 13 significant functions were enriched for the 72 common DEGs at the two time periods. Chemokine (C-C motif) ligand 2 (CCL2), dual-specificity phosphatase-1 (DUSP1) and FAS were associated with the response to GC stimulus and the transcription factor c-Jun bound to promoter regulation regions of CCL2, DUSP1 and FAS. In conclusion, the transcription factors and binding sites of DEGs associated with the response of LECs to GCs may provide potential
\end{abstract}

Correspondence to: Dr Ping Liu, Department of Ophthalmology, The First Affiliated Hospital of Harbin Medical University, 143 Yiman Street, Harbin, Heilongjiang 150001, P.R. China

E-mail: pingliuu@hotmail.com

Key words: lens epithelial cells, glucocorticoid, dexamethasone, function enrichment analysis, transcription factor gene targets for designing and developing drugs to protect against GC-induced cataract formation.

\section{Introduction}

Glucocorticoid (GCs) steroid hormones are used in the treatment of diseases, including rheumatoid arthritis, asthma and various ocular diseases. It has been widely reported that prolonged treatment with GCs can lead to the formation of posterior subcapsular cataracts (1-3). Although numerous attempts have been made to increase understanding of this, the mechanism underlying GC-induced cataract formation remains to be elucidated $(4,5)$.

GCs have important roles in numerous biological processes, including regulation of anti-inflammatory activity and immunosuppressive action $(6,7)$. GCs exert their effects through binding to $\mathrm{GC}$ receptors (GR), which modulate the expression of target genes $(8,9)$. Alternatively, GCs have been proposed to act on the lens indirectly through mechanisms involving oxidative stress and depletion of glutathione $(10,11)$. Global gene profiling was performed to analyze novel GC-induced changes in the gene expression of human lens epithelial cells (LECs) (12). Following this study, pathway analysis was performed in immortalized and primary human LECs and the results demonstrated that GC treatment of LECs activated the GR to modulate the expression of mitogen-activated protein kinase and phosphatidylinositol-3-kinase/AKT regulators (13).

To improve the understanding of the mechanism involved in the formation of cataracts, GC's induction of vascular barrier function requires elucidation. GCs combine with a cytoplasmic receptor that alters gene expression in two ways. One way is dependent on the receptor binding directly to DNA and acts as a transcription factor (positively or negatively). The other is dependent on its binding to and interfering with other transcription factors (14). Transcription factor p54 is essential for GC-mediated expression of occludin, claudin-5 and vascular barrier induction, and the p54/PSF heterodimer may contribute to normal blood-retinal barrier induction in vivo (15). Thus, it is necessary to elucidate the transcription factors that are activated in response to GCs.

The present study aimed to identify differentially expressed genes (DEGs) and their common transcription factors in order to gain a novel insight into the mechanism of action of GCs in LECs. 


\section{Materials and methods}

Affymetrix microarray data. The transcription profile of GSE3040 was obtained from the gene expression omnibus (GEO, http://www.ncbi.nlm.nih.gov/geo/) database, which is based on the GPL96 [HG-U133A] Affymetrix Human Genome U133A Array (Affymetrix Inc., Santa Clara, CA, USA). There were 12 samples of human LECs treated with vehicle or dexamethasone (Dex) at 4 and $16 \mathrm{~h}$. At each time period, there were six samples, of which three samples were treated with vehicle (control group) and three samples were treated with Dex (Dex group). Freshly isolated human LECs were obtained from capsulorhexis specimens following surgery, these were the original cells used in the GEO (12).

Data preprocessing and DEG analysis. The GSE3040 datasets were converted into expression values and pre-processing, including background correction and quartile data normalization were performed using the robust multiarray average algorithm (16) with default parameters in the R language affy package (http://www.bioconductor. org/) $(17,18)$. The linear models for microarray analysis (Limma) package in the R language (www.bioconductor. org/packages/release/bioc/html/limma.html) (19) were used to identify DEGs by performing Student's t-test on the samples. A fold change value $>1$ and $\mathrm{P}<0.05$ were selected as the cut-off criteria.

Hierarchical cluster analysis of DEGs. Gene hierarchical cluster analysis of DEGs was performed using the Pearson correlation coefficient algorithm (20) in cluster 3.0 (21).

Functional enrichment analysis of common DEGs. The Database for Annotation, Visualization and Integrated Discovery (DAVID; http://david.abcc.Ncifcrf.gov/) (22), a high-throughput and integrated data-mining environment, analyzes gene lists derived from high-throughput genomic experiments. After the common DEGs were selected, DAVID was used to identify over-represented gene ontology (GO; http://www. geneontology. org/) categories in biological processes based on the hypergeometric distribution. The GO terms with a value of $\mathrm{P}<0.05$ were selected as significantly enriched DEGs.

Transcription factors and binding site analysis. A transcription factor is a protein, which binds to specific DNA sequences. The TRANSFAC database comprising information about transcription factors, target genes and binding sites has been developed (23). The TRANSFAC database was used to screen transcription factors and binding sites on DEGs in response to GCs.

\section{Results}

$D E G$ analysis. The publicly available microarray dataset, GSE3040, was obtained from the GEO database. Student's t-test was used to identify genes specifically differentially expressed at 4 and $16 \mathrm{~h}$ with the cut-off criteria of $\mathrm{P}<0.05$ and fold change $>1$. The results revealed that 696 and 949 genes at 4 and $16 \mathrm{~h}$, respectively, exhibited significant differential expression.

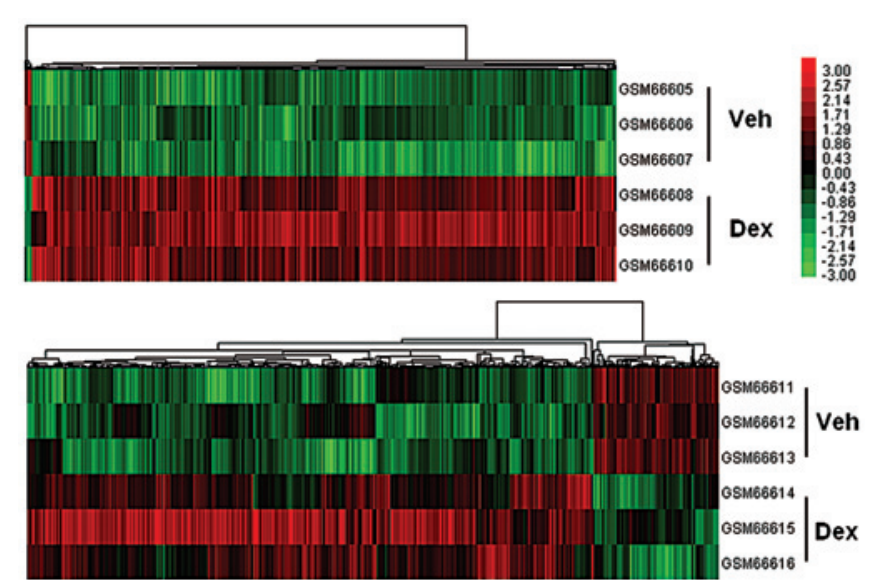

Figure 1. Heat map of cluster analysis of differentially expressed genes between Veh and Dex samples. Green, downregulated genes; and Red, upregulated genes. Dex, dexamethasone; veh, vehicle.

Hierarchical cluster analysis of DEGs between Veh and Dex samples at two time periods. As indicated using hierarchical cluster analysis, the expression levels of DEGs were markedly increased in Veh samples compared with that of the Dex group, at 4 and $16 \mathrm{~h}$ (Fig. 1).

Set comparison of DEGs between two time periods. DEGs set at 4 and $16 \mathrm{~h}$ were compared and presented as a Venn diagram (Fig. 2A). There were 72 common DEGs. The expression folds of 72 DEGs at 4 and $16 \mathrm{~h}$ are shown in Fig. 2B. The results revealed that the gene expression trend at $4 \mathrm{~h}$ was the same as that at $16 \mathrm{~h}$.

GO enrichment analysis. To gain further insight into the function of genes in our interaction network, the online biological classification tool DAVID was used. A total of 13 significant GO function enrichment nodes were obtained and the distributions of genes is shown in Fig. 3. As Table I demonstrates, Chemokine (C-C motif) ligand 2 (CCL2), dualspecificity phosphatase-1 (DUSP1) and FAS were associated with the function of response to GC stimulus ( $\mathrm{P}=0.0496906$ ). Expression of CCL2 was downregulated, and DUSP1 and FAS were upregulated at 4 and $16 \mathrm{~h}(\mathrm{P}<0.05)$.

Transcription factor analysis. Using the TRANSFAS database, the transcription factors and binding sites, which were associated with the three GC response genes, CCL2, DUSP1 and FAS, were assessed. As Fig. 4 demonstrates, c-Jun binds to the promoter regulatory regions of these three genes and was the common transcription factor (Fig. 4).

\section{Discussion}

GCs have been used in clinical treatment for decades; however, prolonged GC treatment may lead to the formation of cataracts (24). In the current study, using DNA microarray analysis, the gene expression profiles of human LECs treated with Dex or vehicle were analyzed. A total of 13 significant GO functions were identified and CCL2, DUSP1 and FAS genes were associated with a response to GC stimulus. The transcription factor that binds to CCL2, DUSP1 and FAS 
Table I. Enriched Gene Ontology terms of the common differentially expressed genes at $4 \mathrm{~h}$ and $16 \mathrm{~h}(\mathrm{P}<0.05)$.

\begin{tabular}{|c|c|c|c|c|}
\hline Term & Function & Count & P-value & Genes \\
\hline GO:0048545 & Response to steroid hormone stimulus & 7 & 0.000240124 & $\begin{array}{l}\text { KCNMA1, CCL2, DUSP1, } \\
\text { LEPR, ESR1, FAS, CD24 }\end{array}$ \\
\hline GO:0009725 & Response to hormone stimulus & 9 & 0.000255726 & $\begin{array}{l}\text { KCNMA1, CCL2, DUSP1, } \\
\text { LEPR, ESR1, FOXC2, } \\
\text { FAS, CD24, STAT1 }\end{array}$ \\
\hline GO:0009719 & Response to endogenous stimulus & 9 & 0.000494549 & $\begin{array}{l}\text { KCNMA1, CCL2, DUSP1, } \\
\text { LEPR, ESR1, FOXC2, } \\
\text { FAS, CD24, STAT1 }\end{array}$ \\
\hline GO:0042981 & Regulation of apoptosis & 12 & 0.000955092 & $\begin{array}{l}\text { KCNMA1, PRUNE2, CCL2, } \\
\text { DUSP1, MCL1, SOS2, } \\
\text { ESR1, FOXC2, FAS, } \\
\text { CD24, STAT1, ANGPTL4 }\end{array}$ \\
\hline GO:0043067 & Regulation of programmed cell death & 12 & 0.001035716 & $\begin{array}{l}\text { KCNMA1, PRUNE2, CCL2, } \\
\text { DUSP1, MCL1, SOS2, } \\
\text { ESR1, FOXC2, FAS, CD24, } \\
\text { STAT1, ANGPTL4 }\end{array}$ \\
\hline GO:0010941 & Regulation of cell death & 12 & 0.001067374 & $\begin{array}{l}\text { KCNMA1, PRUNE2, CCL2, } \\
\text { DUSP1, MCL1, SOS2, } \\
\text { ESR1, FOXC2, FAS, } \\
\text { CD24, STAT1, ANGPTL4 }\end{array}$ \\
\hline GO:0043627 & Response to estrogen stimulus & 5 & 0.001353113 & $\begin{array}{l}\text { KCNMA1, DUSP1, LEPR, } \\
\text { ESR1, CD24 }\end{array}$ \\
\hline GO:0010033 & Response to organic substance & 10 & 0.005300596 & $\begin{array}{l}\text { KCNMA1, CCL2, DUSP1, } \\
\text { MCL1, LEPR, ESR1, } \\
\text { FOXC2, FAS, CD24, STAT1 }\end{array}$ \\
\hline GO:0031960 & Response to corticosteroid stimulus & 4 & 0.006933831 & $\begin{array}{l}\text { KCNMA1, CCL2, DUSP1, } \\
\text { FAS }\end{array}$ \\
\hline GO:0043065 & Positive regulation of apoptosis & 7 & 0.013655682 & $\begin{array}{l}\text { KCNMA1, PRUNE2, DUSP1, } \\
\text { SOS2, FAS, CD24, STAT1 }\end{array}$ \\
\hline GO:0043068 & $\begin{array}{l}\text { Positive regulation of programmed } \\
\text { cell death }\end{array}$ & 7 & 0.01409138 & $\begin{array}{l}\text { KCNMA1, PRUNE2, DUSP1, } \\
\text { SOS2, FAS, CD24, STAT1 }\end{array}$ \\
\hline GO:0010942 & Positive regulation of cell death & 7 & 0.01438722 & $\begin{array}{l}\text { KCNMA1, PRUNE2, DUSP1, } \\
\text { SOS2, FAS, CD24, STAT1 }\end{array}$ \\
\hline GO:0051384 & Response to glucocorticoid stimulus & 3 & 0.049690594 & CCL2, DUSP1, FAS \\
\hline
\end{tabular}

CCL2, chemokine (C-C motif) ligand 2; DUSP1, dual specificity protein phosphatase 1.

A

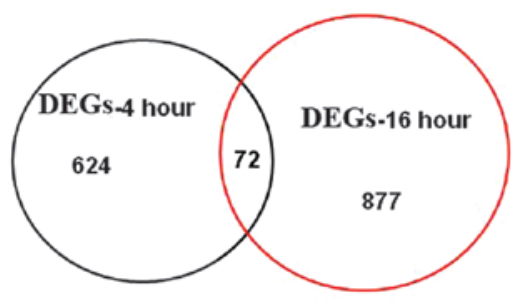

B

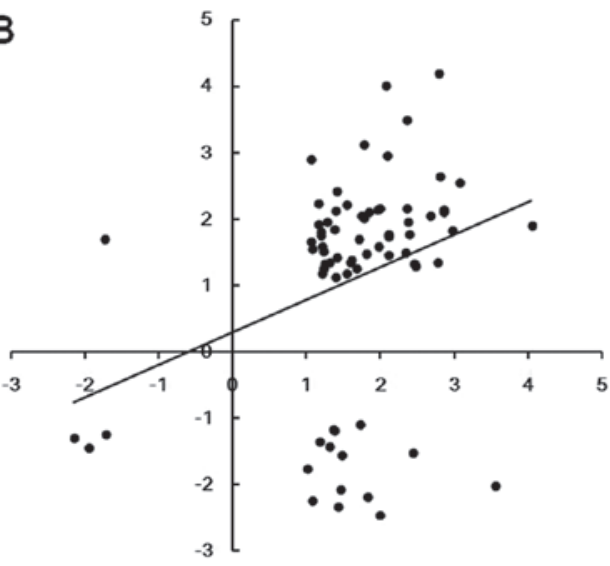

Figure 2. DEGs in lens epithelial cells in response to dexamethasone. (A) Venn diagram depicting common DEGs. The black and red circles represent DEGs at $4 \mathrm{~h}$ and $16 \mathrm{~h}$ treated with dexamethasone, respectively. (B) Correlation of gene expression of common DEGs between $4 \mathrm{~h}$ and $16 \mathrm{~h}$. X-axis, log(FC) of DEGs at $4 \mathrm{~h}$; and Y-axis, $\log$ (FC) of DEGs at $16 \mathrm{~h}$. DEGs, differentially expressed genes; FC, fold change. 


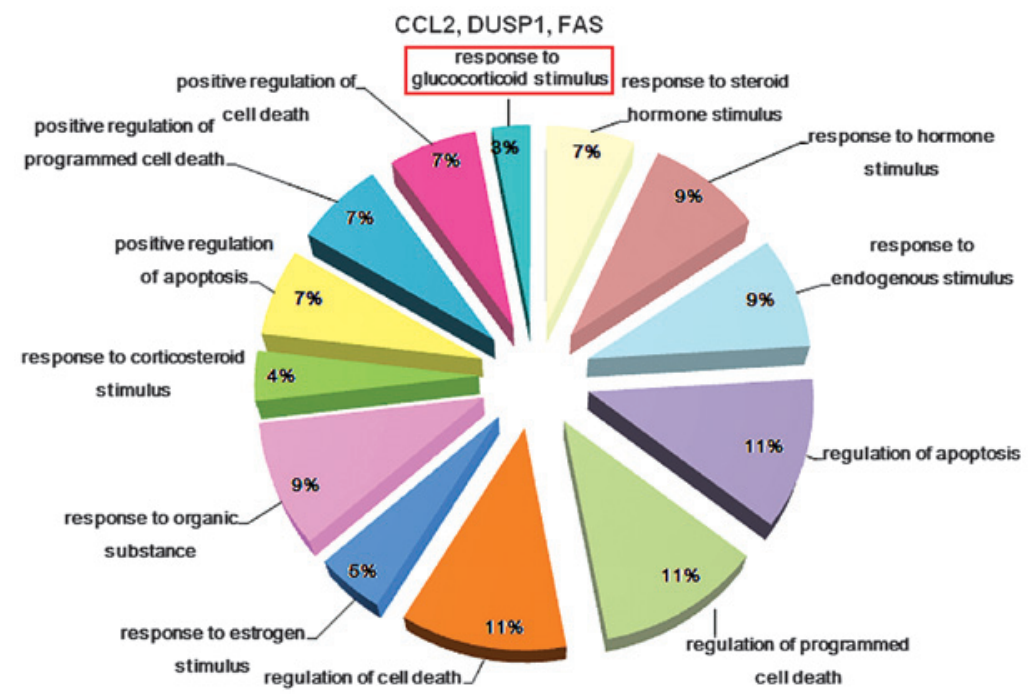

Figure 3. Pie chart depicting the hierarchical clustering of enriched functions involving common differentially expressed genes in the lens epithelial cells treated with dexamethasone.

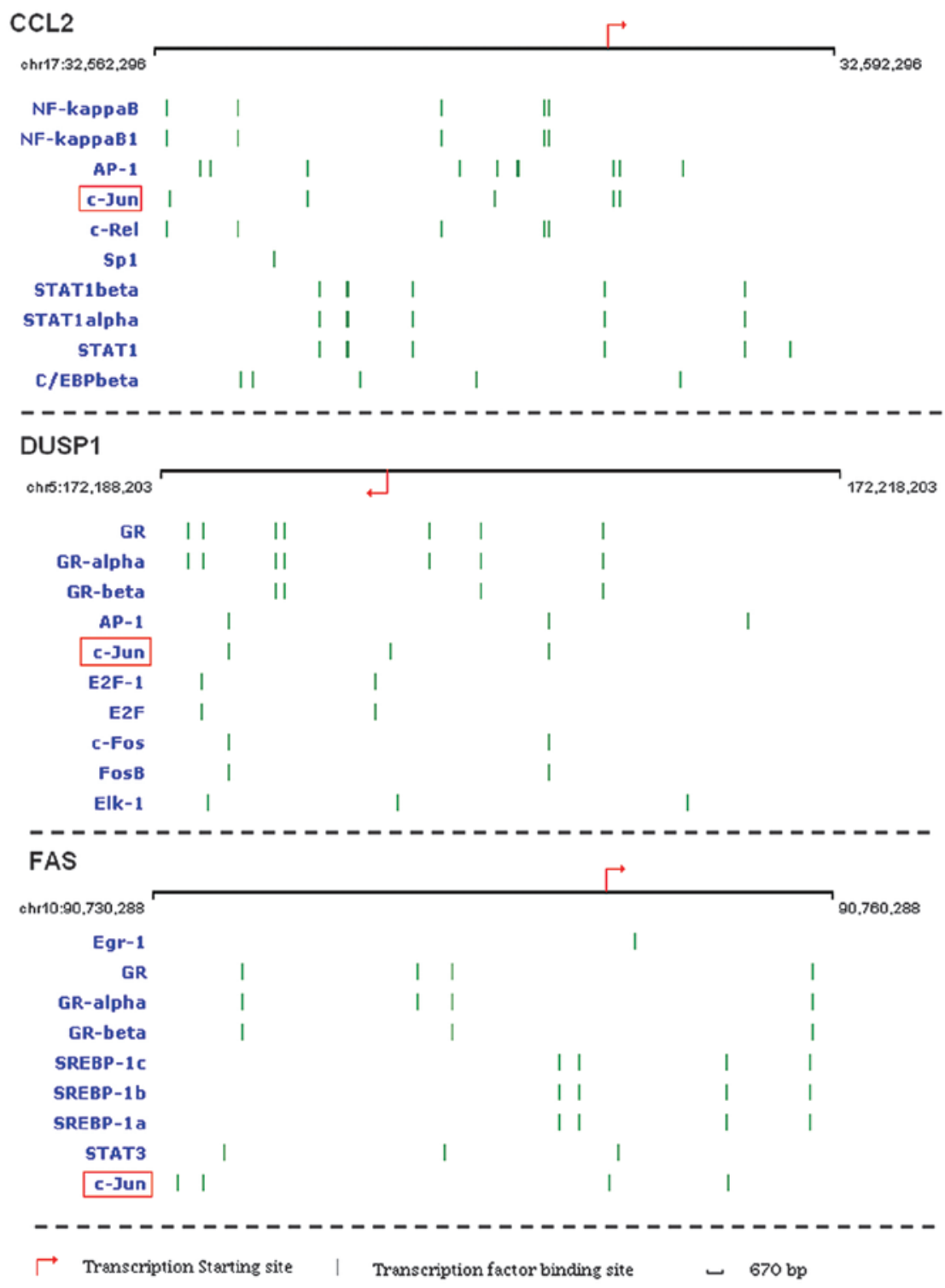

Figure 4. Map of transcription factor binding on the promoter regulatory regions of CCL2, DUSP1 and FAS. Green bars represent the binding sites. Red arrows represent transcription initiation sites and directions. CCL2, chemokine (C-C motif) ligand 2; DUSP1, dual specificity protein phosphatase 1. 
were also analyzed. The results demonstrated that c-Jun was a common transcription factor between these genes.

CCL2 is also known as monocyte chemotactic protein-1 and is secreted by endothelial cells, fibroblasts and monocytes (25). It has been reported that CCL2 expression and macrophage accumulation were inhibited by treatment with Dex in cholesterol-fed rabbits (26). GRs may bind specifically to CCL2 mRNA and the inflammatory response of the GR was mediated by regulation of CCL2 mRNA stability (27). CCL2 was detected in the sample obtained from patients following cataract surgery (28). DUSP1 is a member of the threonine-tyrosine dual-specificity phosphatases (29). Increased expression of GILZ mRNA and DUSP1 mRNA and protein was observed in immortalized and donor immortalized primary LECs (13). The induction of DUSP1 is dependent on the GR and typically occurs within $\leq 1 \mathrm{~h}(30)$. The FAS receptor is an important cell surface receptor protein of the tumor necrosis factor receptor family (31). Yang et al (32) reported that FAS ligand expression was inhibited by retinoic acid and GCs.

In the present study, c-Jun was observed to bind the promoter regulatory regions of CCL2, DUSP1 and FAS. The c-Jun gene encodes a basic region-leucine zipper transcription factor implicated in numerous cellular processes. C-Jun regulates gene expression and cell function by being involved in the formation of a variety of dimeric complexes, which exhibit high affinity sequence specific DNA-binding activity (33). It has been reported that c-Jun attenuated MG132-induced activation of activator protein-1 and expression of CCL2 (34). The Hepatitis $\mathrm{C}$ virus core protein expression activated MAP kinase phosphatase, increased DUSP1 expression and increased cell proliferation, which was accompanied by an activation of c-Jun (35). The expression of dominant-negative c-Jun in melanoma cells efficiently increased Fas expression (36). The present results demonstrated that c-Jun may be the critical transcription factor, which affected gene expression in LECs in response to GCs.

In conclusion, the gene expression profiles of LECs following GC treatment were analyzed using bioinformatics analysis and it was found that CCL2, DUSP1 and FAS are involved in the response to GC stimulus. The transcription factor c-Jun, when bound to CCL2, DUSP1 and FAS, may affect their expression. CCL2, DUSP1, FAS and transcription factor c-Jun may be used as specific therapeutic molecular targets in order to treat cataracts induced by GCs. However, further studies are required to confirm the present results.

\section{Acknowledgements}

The authors would like to thank the Laboratory of Medical Genetics, Harbin Medical University, Harbin, China for the technical assistance that was required. The authors would also to thank Fenghe (Shanghai) Information Technology Co., Ltd. Their input and assistance provided a valuable added dimension to the present study.

\section{References}

1. Black RL, Oglesby RB, von Sallmann L and Bunim JJ: Posterior subcapsular cataracts induced by corticosteroids in patients with rheumatoid arthritis. JAMA 174: 166-171, 1960.
2. Williamson J, Paterson RW, McGavin DD, Jasani MK, Boyle JA and Doig WM: Posterior subcapsular cataracts and glaucoma associated with long-term oral corticosteroid therapy. In patients with rheumatoid arthritis and related conditions. Br J Ophthalmol 53: 361-372, 1969.

3. Urban RC Jr and Cotlier E: Corticosteroid-induced cataracts. Surv Ophthalmol 31: 102-110, 1986.

4. Jobling AI and Augusteyn RC: What causes steroid cataracts? A review of steroid-induced posterior subcapsular cataracts. Clin Exp Optom 85: 61-75, 2002.

5. Auphan N, DiDonato JA, Rosette C, Helmberg A and Karin M: Immunosuppression by glucocorticoids: inhibition of NF- $\mathrm{KB}$ activity through induction of $\mathrm{I} \kappa \mathrm{B}$ synthesis. Science 270: 286-290, 1995.

6. Ehrchen J, Steinmüller L, Barczyk K, et al: Glucocorticoids induce differentiation of a specifically activated, anti-inflammatory subtype of human monocytes. Blood 109: 1265-1274, 2007.

7. Reichardt HM, Tronche F, Berger S, Kellendonk C and Schütz G: New insights into glucocorticoid and mineralocorticoid signaling: lessons from gene targeting. Adv Pharmacol 47: 1-21, 2000.

8. Aranda A and Pascual A: Nuclear hormone receptors and gene expression. Physiol Rev 81: 1269-1304, 2001.

9. Webster JC and Cidlowski JA: Mechanisms of glucocorticoid-receptor-mediated repression of gene expression. Trends Endocrinol Metab 10: 396-402, 1999.

10. Nishigori H, Lee JW, Yamauchi Y and Iwatsuru M: The alteration of lipid peroxide in glucocorticoid-induced cataract of developing chick embryos and the effect of ascorbic acid. Curr Eye Res 5: 37-40, 1986.

11. Lou MF, Dickerson JE Jr, Garadi R and York BM Jr: Glutathione depletion in the lens of galactosemic and diabetic rats. Exp Eye Res 46: 517-530, 1988.

12. Gupta V, Galante A, Soteropoulos P, Guo S and Wagner BJ: Global gene profiling reveals novel glucocorticoid induced changes in gene expression of human lens epithelial cells. Mol Vis 11: 1018-1040, 2005.

13. Gupta V, Awasthi N and Wagner BJ: Specific activation of the glucocorticoid receptor and modulation of signal transduction pathways in human lens epithelial cells. Invest Ophthalmol Vis Sci 48: 1724-1734, 2007.

14. Saklatvala J: Glucocorticoids: do we know how they work? Arthritis Res 4: 146-150, 2002.

15. Keil JM, Liu X and Antonetti DA: Glucocorticoid induction of occludin expression and endothelial barrier requires transcription factor p54 NONO. Invest Ophthalmol Vis Sci 54: 4007-4015, 2013

16. Best CJ, Gillespie JW, Yi Y, et al: Molecular alterations in primary prostate cancer after androgen ablation therapy. Clin Cancer Res 11: 6823-6834, 2005.

17. Team RC: R: A language and environment for statistical computing. Vienna, Austria: R Foundation for Statistical Computing : 1-1731, 2008.

18. Gautier L, Cope L, Bolstad BM and Irizarry RA: affy - analysis of Affymetrix GeneChip data at the probe level. Bioinformatics 20: 307-315, 2004.

19. Reimers M and Carey VJ: Bioconductor: an open source framework for bioinformatics and computational biology. Methods Enzymol 411: 119-134, 2006.

20. Eisen MB, Spellman PT, Brown PO and Botstein D: Cluster analysis and display of genome-wide expression patterns. Proc Natl Acad Sci USA 95: 14863-14868, 1998.

21. Sarle WS: Algorithms for clustering data. Technometrics 32: 227-229, 1990.

22. Huang da W, Sherman BT and Lempicki RA: Systematic and integrative analysis of large gene lists using DAVID bioinformatics resources. Nat Protoc 4: 44-57, 2009.

23. Matys V, Fricke E, Geffers R, et al: TRANSFAC: transcriptional regulation, from patterns to profiles. Nucleic Acids Res 31: 374-378, 2003

24. Cumming RG, Mitchell $P$ and Leeder SR: Use of inhaled corticosteroids and the risk of cataracts. N Engl J Med 337: 8-14, 1997.

25. Gu L, Tseng SC and Rollins BJ: Monocyte chemoattractant protein-1. Chem Immunol 72, 7-29, 1999.

26. Poon M, Gertz SD, Fallon JT, et al: Dexamethasone inhibits macrophage accumulation after balloon arterial injury in cholesterol fed rabbits. Atherosclerosis 155: 371-380, 2001.

27. Dhawan L, Liu B, Blaxall BC and Taubman MB: A novel role for the glucocorticoid receptor in the regulation of monocyte chemoattractant protein-1 mRNA stability. J Biol Chem 282: 10146-10152, 2007. 
28. Janciauskiene S, Westin K, Grip O and Krakau T: Detection of Alzheimer peptides and chemokines in the aqueous humor. Eur J Ophthalmol 21: 104-111, 2011.

29. Boutros T, Chevet E and Metrakos P: Mitogen-activated protein (MAP) kinase/MAP kinase phosphatase regulation: roles in cell growth, death, and cancer. Pharmacol Rev 60: 261-310, 2008.

30. Abraham SM, Lawrence T, Kleiman A, et al: Antiinflammatory effects of dexamethasone are partly dependent on induction of dual specificity phosphatase 1. J Exp Med 203: 1883-1889, 2006

31. Nagata S: Fas and Fas ligand: a death factor and its receptor. Adv Immunol 57: 129-144, 1994.

32. Yang Y, Merćep M, Ware CF and Ashwell JD: Fas and activation-induced Fas ligand mediate apoptosis of $\mathrm{T}$ cell hybridomas: inhibition of Fas ligand expression by retinoic acid and glucocorticoids. J Exp Med 181: 1673-1682, 1995.
33. Wisdom R, Johnson RS and Moore C: c-Jun regulates cell cycle progression and apoptosis by distinct mechanisms. EMBO J 18: 188-197, 1999.

34. Nakayama K, Furusu A, Xu Q, Konta T and Kitamura M: Unexpected transcriptional induction of monocyte chemoattractant protein 1 by proteasome inhibition: involvement of the c-Jun N-terminal kinase-activator protein 1 pathway. J Immunol 167: 1145-1150, 2001.

35. Erhardt A, Hassan M, Heintges T and Häussinger D: Hepatitis C virus core protein induces cell proliferation and activates ERK, JNK, and p38 MAP kinases together with the MAP kinase phosphatase MKP-1 in a HepG2 Tet-Off cell line. Virology 292: 272-284, 2002.

36. Herdegen T, Claret FX, Kallunki T, et al: Lasting N-terminal phosphorylation of c-Jun and activation of c-Jun N-terminal kinases after neuronal injury. J Neurosci 18: 5124-5135, 1998. 